\title{
Análisis Descriptivo de un Modelo de Osteoporosis Inducida en Ratas (Rattus norvegicus)
}

\author{
Descriptive Analysis of an Induced Osteoporosis Model in Rats (Rattus norvegicus)
}

\author{
Marcelo Parra ${ }^{1,2}$; Ingrid Romero ${ }^{1,3}$; Sergio Olate ${ }^{1,4}$
}

PARRA, M.; ROMERO, I. \& OLATE, S. Análisis descriptivo de un modelo de osteoporosis inducida en ratas (Rattus norvegicus). Int. J. Morphol., 39(1):282-286, 2021.

RESUMEN: El objetivo del presente estudio fue realizar un análisis histopatológico de un modelo animal en ratas, que simule las características observables en huesos de pacientes con osteoporosis. Para ello, se utilizaron 10 ratas hembras (Rattus norvegicus) Sprague Dawley de 12 semanas de desarrollo y aproximadamente 200-250 g. De las cuales, a 5 se le realizó ovariectomía (OVX) bilateral, mientras que las 5 restantes fueron utilizadas como control. Posterior a 12 semanas de realizadas las ovariectomías en el grupo experimental, se realizó la eutanasia de los animales y la obtención de ambos fémur, los cuales fueron posteriormente seccionados para procesar su porción distal para su procesamiento histológico de rutina. Todos los animales sobrevivieron al final del estudio sin ninguna complicación postoperatoria, las imágenes histológicas evidenciaron en el grupo experimental (OVX), una disminución del grosor del hueso cortical, mayor cantidad de hueso esponjoso, pérdida de la continuidad de periostio y endostio alrededor de la matriz ósea además de mayor cantidad de tejido adiposo en la médula ósea, al ser comparados con el grupo control. Se puede concluir que a las 12 semanas post ovariectomía se observa un fenotipo histopatológico compatible con características oseteoporóticas en ratas adultas.

PALABRAS CLAVE: Osteoporosis; Ovariectomía; Modelo animal.

\section{INTRODUCCIÓN}

La osteoporosis, es una enfermedad generalizada del sistema esquelético caracterizada por la pérdida de masa ósea y por eldeterioro de la microarquitectura del tejido óseo, que compromete la resistencia y determina una mayor fragilidad y susceptibilidad a fracturas (Klibanski et al., 2001). Dentro de las causas descritas, destacan principalmente la incapacidad de producir un esqueleto de masa y fuerza óptima durante el crecimiento, reabsorción ósea excesiva y una respuesta de formación inadecuada al aumento de la reabsorción durante la remodelación ósea. (Klibanski et al., 2001). De esta forma, el envejecimiento y los cambios hormonales juegan un rol crítico en el desarrollo de las últimas 2 causas mencionadas. (Raisz, 2005)
Respecto al envejecimiento, la literatura indica que el hueso cortical se vuelve más frágil y débil con la edad. (Gustafsson et al., 2019, Kim et al., 2020b) De manera similar el hueso medular o esponjoso también se debilita con la edad. (Westbury et al., 2020) Por otro lado, cambios hormonales también juegan un papel preponderante en el desarrollo de la Osteoporosis, siendo el Estrógeno una de las hormónas mas importantes. Estudios morfológicos y mediciones de determinados marcadores bioquímicos han indicado que la remodelación ósea se acelera en la menopausia. El aumento de la formación ósea que normalmente se produce en respuesta a la carga mecánica disminuye en relación a la deficiencia de estrógenos, lo que sugiere que el estrógeno es anti catabólico y anabólico. (Raisz, 2005)

\footnotetext{
${ }^{1}$ Programa de Doctorado en Ciencias Morfológicas, Centro de Excelencia en Estudios Morfológicos y Quirúrgicos (CEMyQ), Facultad de Medicina, Universidad de La Frontera, Temuco, Chile.

${ }^{2}$ Departamento de Odontología Integral Adultos, Facultad de Odontología, Universidad de La Frontera, Temuco, Chile.

${ }^{3}$ Departamento de Procesos Diagnósticos y Evaluación, Carrera de Nutrición, Facultad Ciencias de la Salud, Universidad Católica de Temuco, Temuco, Chile.

${ }^{4}$ División de Cirugía Oral, Facial y Maxilofacial, Facultad de Odontología, Universidad de La Frontera, Temuco, Chile.
} 
La osteoporosis no solo afecta a los huesos de la cadera y huesos largos, si no también a los huesos de la cara, entre ellos lamandíbula, en la cual, estudios han mostrado disminuciones en la altura del reborde alveolar, densidad del hueso trabecular o medular además del grosor de la cortical mandibular.

Muchos son los procedimientos quirúrgicos en el área craneofacial que requieren una respuesta eficiente del tejido óseo lo que se puede alterar en pacientes con Osteoporosis. Debido a esto, es necesario el desarrollo de modelos animales con la finalidad de no solo evaluar parámetros clínicos de diferentes procedimientos quirúrgicos, si no también comprender a nivel histológico y molecular, como un hueso con osteoporosis reacciona ante distintos procedimientos, como instalación implantes dentales, injertos óseos, reducción de fracturas, entre otros.

En modelos animales, específicamente en ratas, se plantea que posterior a las 6-8 semanas, luego de la extirpación de los ovarios, se evidenciaría un estado de osteoporosis por déficit de estrógeno (Chen et al., 2018; Liu et al., 2018; Mohamed et al., 2018; Xu et al., 2018; Shimizu et al., 2018.). No obstante, aun no existe consenso respecto al tiempo necesario post OVX para obtener rasgos fenotípicos claros de osteoporosis.

El objetivo del presente estudio fue realizar un análisis histopatológico de un modelo animal que simule las características observables en huesos de pacientes con osteoporosis.

\section{MATERIAL Y MÉTODO}

En el presente estudio, se utilizaron 10 ratas hembras (Ratus norvegicus) Sprague Dawley de 12 semanas de desarrollo y aproximadamente 200-250 g. Los animales fueron obtenidos de manera aleatoria por el encargado del Bioterio del Centro de Excelencia en Estudios Morfológicos y Quirúrgicos (CEMyQ) de la Universidad de La Frontera, Temuco, Chile, quien no participó del presente estudio, ubicados en jaulas separadas con acceso libre a agua y comida (ad libitum), con temperatura ambiente de $20 \pm 2{ }^{\circ} \mathrm{C}$ y ciclos de luz/oscuridad de 12 horas. Todos los procedimientos de manejo de los animales fueron llevados a cabo siguiendo las directrices establecidas en la Guía para el Cuidado y Uso de los Animales de Laboratorio (2011), y aprobado por el Comité de Ética Científico de la Universidad de La Frontera. N004_19.

Todos los procedimientos quirúrgicos fueron realizados en la sala de Cirugía Experimental del CEMyQ, bajo supervisión ycolaboración del Médico Veterinario a car- go. Cinco de las 10 ratas fueron sometidas a ovariectomía bilateral para generar un estado de osteoporosis inducida por déficit de estrógeno y las 5 restantes utilizadas como control.

Ovariectomía. Todas las ratas del grupo experimental fueron sometidas a ovariectomía bilateral previo anestesia general con Ketamina $80 \mathrm{mg} / \mathrm{Kg}$ y Xilacina $10 \mathrm{mg} / \mathrm{Kg}$. Posterior a la anestesia, fue rasurada la región cutánea del abdomen con una máquina eléctrica MOSER $®$ y posteriormente aplicación de povidona yodada (MDK S.A.) en la zona donde se realizará la incisión. Bajo un campo operatorio estéril, se realizó la incisión y acceso de la zona de interés. Posterior a la localización de los ovarios, éstos fueron pinzados en su parte inferior con una pinza Kelly, luego, se realizó una ligadura en la zona inferior del pinzamiento para evitar hemorragias y posterior a eso se realizó la extirpación del el ovario propiamente tal, finalmente se realizó la sutura plano por plano, con sutura reabsorbible, (Ácido poligligólico) 4.0 en las zonas mas profundas, y seda 3.0 en la piel. Como control postoperatorio, se administró Flunixin meglumine: $1,1 \mathrm{mg}$ /Kg vía intramuscular cada 24 horas por 3 a 4 días.

Posterior a 12 semanas, se realizó la eutanasia de los animales mediante sobredosis de Tiopental sódico, y se realizo la obtención de ambos fémur, el cual fue posteriormente seccionado para procesar su porción distal.

Procesamiento y análisis histológico. Inmediatamente después de extraídas, las muestras fueron sumergidas en formalina tamponada al $10 \%$ para su fijación por 48 horas, posterior a eso, fueron descalcificadas en EDTA tamponado al $10 \%$ por dos a tres meses, deshidratados en una batería de alcoholes ascendentes e incluidos en Paraplast (Histosec $\AA$ pastilles, Merck Millipore, Darmstadt, Alemania). Los bloques de parafina fueron confeccionados y cortados a $6 \mathrm{~mm}$ de grosor con disposición proximo-distal, en un micrótomo (Leica® ${ }^{\circledR}$ RM2255, Wetzlar, Alemania), teñidos con: Hematoxilina \& Eosina para su análisis histológico. Todas las muestran fueron analizadas bajo microscopía de luz. El análisis descriptivo de las muestras fue realizado empleando microscopia de luz (Leica® DM750, Wetzlar, Alemania).

\section{RESULTADOS}

Todos los animales sobrevivieron al final del estudio sin ninguna complicación postoperatoria durante las 12 semanas post ovariectomía, 10 fémures fueron analizados bajo microscopía de luz. 
Las imágenes de microscopía de luz, mostraron en el grupo control una cortical formada por hueso compacto de grosor adecuado, observándose los complejos de osteonas en ella, con sus respectivos canales centrales y laterales en donde se aprecia la irrigación correspondiente, además de cada canal central rodeado por matriz ósea en donde los osteocitos se disponen formando láminas alrededor de él, característica clásica del hueso laminillar. (Fig. 1a) Mientras que desde la cortical hacia el centro del hueso es posible observar hueso esponjoso de manera conservada. Periostio y endostio bien conservado formado por tejido conectivo denso en donde es posible encontrar osteoblastos y células de revestimiento óseo. Médula ósea con alta celularidad de tipo hematopoyética. (Fig. 1b)

Por otro lado, en el grupo OVX, en la cortical del hueso es posible observar una disminución considerable en el grosor de la misma. El hueso comienza a ser reemplazado por hueso esponjoso.(Fig 2a) El complejo de osteona ya no es posible de observar de manera tan organizada y los osteocitos van perdiendo su organización concéntrica alre-

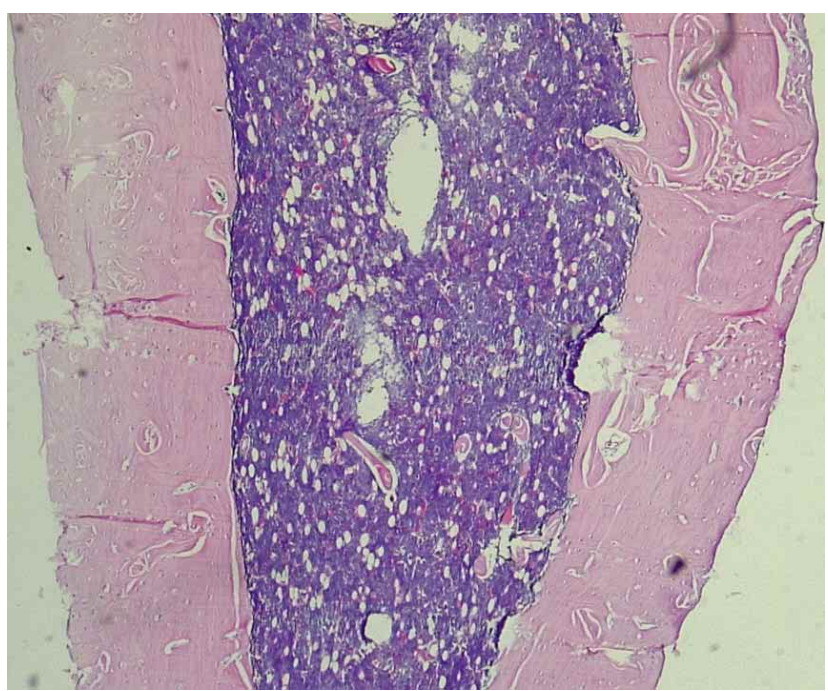

Fig. 1a. Corte histológico de fémur de rata, tinción H\&E, aumento $4 \mathrm{x}$.

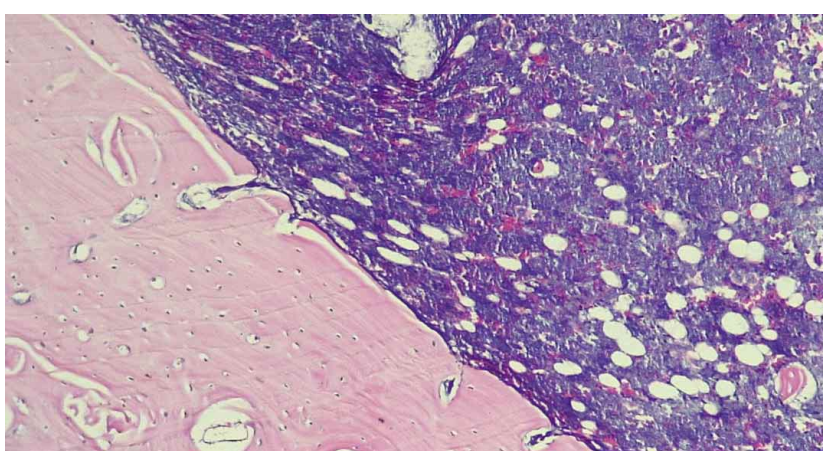

Fig. 1b. Corte histológico de fémur de rata, tinción H\&E, aumento 10x. dedor de los canales centrales de las osteonas. Periostio y endostio pierden su continuidad alrededor de la matriz ósea. Hacia el centro del hueso, es posible observar hueso esponjoso con un trabeculado óseo muy desorganizado. La médula ósea presenta menor cantidad de tejido hematopoyético y es posible observar mayor cantidad de tejido adiposo de tipo unicelular o blanco. Existe una evidente desorganización celular. (Fig. 2b).

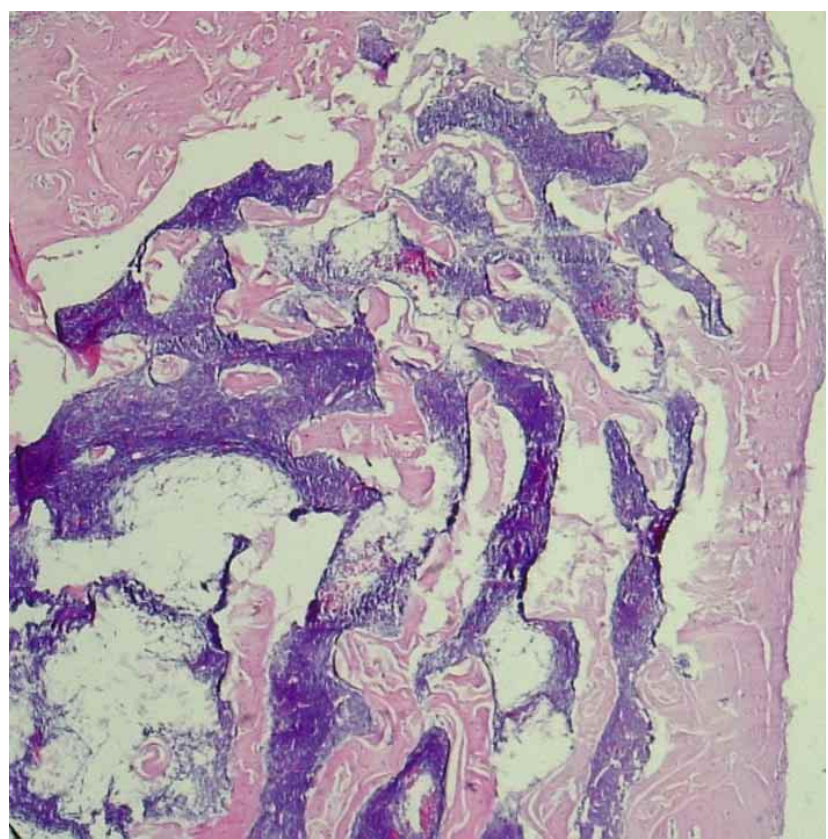

Fig. 2a. Corte histológico de fémur de rata, tinción H\&E, aumento $4 \mathrm{x}$.

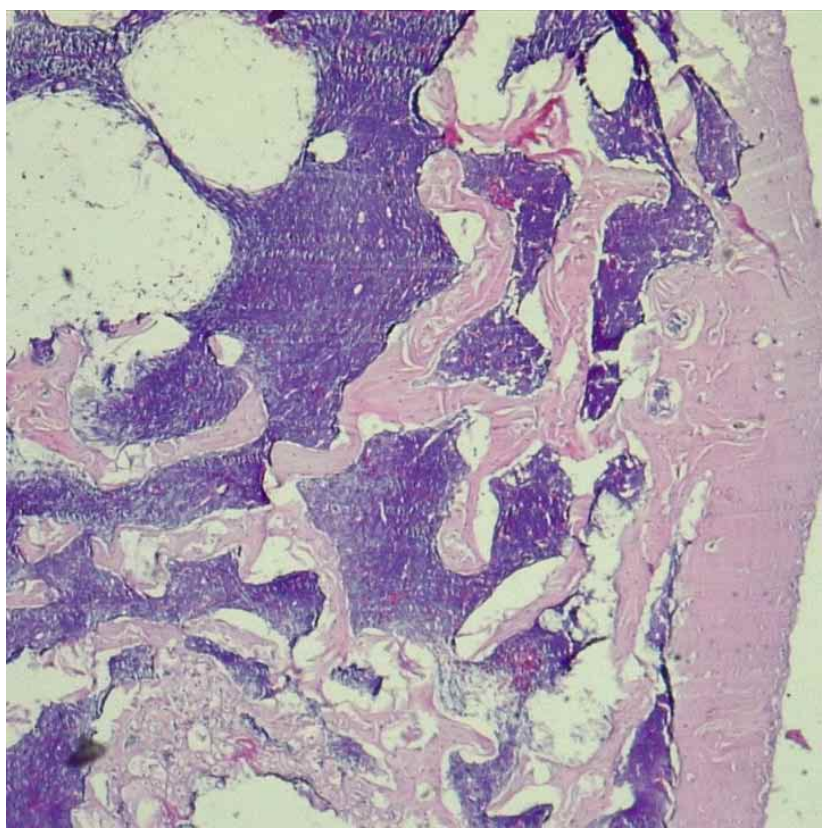

Fig. 2b. Corte histológico de fémur de rata, tinción H\&E, aumento $10 \mathrm{x}$. 


\section{DISCUSIÓN}

El objetivo del presente estudio fue evaluar histopatológicamente el desarrollo de un modelo animal que simule las características observables en huesos osteoporóticos. Existen distintos modelos que buscar emular los efectos de la osteoporosis en el hueso, tales como, utilización de animales envejecidos, baja ingesta de calcio en la dieta, inducción por déficit de estrógeno mediante ovariectomía, entre otros. (Silveira et al., 2020, Tang et al., 2019)

El desarrollo de modelos por OVX ha cobrado relevancia, debido a que permite aislar la variable envejecimiento al momento de realizar el estudio. El modelo por OVX se sustenta en la base de que la deficiencia de estrógeno, el cual produciría un aumento en la reabsorción por parte de los osteoclastos y una respuesta de formación ósea deficiente. (Raisz, 2005) Dicho modelo ha sido utilizado en cirugía oral para estudiar la relación de la osteoporosis en reparación ósea en alveolos post extracción, en la interacción con diferentes materiales de relleno óseo, así como también con la instalación de implantes dentales, observándose resultados diversos. (Chen et al., 2018, Park et al., 2020)

Si bien el modelo de OVX se utiliza bastante, no todos los estudios realizan una confirmación de la obtención del fenotipo osteoprótico, y aquellos estudios que si lo han realizado, utilizan principalmente imagenología 3D en huesos largos para identificar las características anatómicas y la relación de hueso trabecular con hueso cortical. Chen et al., 2018, en un estudio en ratas, mediante imagenología 3D identificaron que a las 8 semanas post ovariectomía la porción distal del fémur reveló una disminución significativa del volumen trabecular al ser comparadas con un grupo sin ovariectomía. Wei et al., 2016 por su parte, en un estudio similar en ratas, detectaron mediante Densitometría Ósea (BMD) diferencias significativas a las 12 semanas entre el grupo OVX y el grupo control, siendo mayores los valores obtenidos en el grupo OVX. Los resultados del presente estudio confirman lo indicado previamente, toda vez que nuestras imágenes logran evidenciar una disminución de la cortical ósea del fémur, siendo ésta reemplazada progresivamente por hueso trabecular desorganizado en el grupo OVX a las 12 semanas.

La osteoporosis, junto con una disminución de la masa ósea y alteración de la microarquitectura ósea, también se asocia con un aumento de la adipogénesis medular, y una disminución de la osteoblastogénesis (Li et al., 2016) lo cual se conoce como la "teoría de la grasa de la osteoporosis" En la cual, el microambiente de la médula ósea cambia progresivamente en condiciones patológicas vinculadas a la osteoporosis y se caracteriza por un aumento del estrés oxidativo, inflamación crónica, señales osteogénicas suprimidas e inhibidores osteoblásticos elevados. Estos factores ambientales patológicos contribuyen a un cambio en la diferenciación de las células mesenquimales, favoreciendo la diferenciación hacia adipocitos sobre los osteoblastos, aumentando por consiguiente la cantidad de tejido adiposo en la médula ósea. (Li et al., 2016) Los resultados del presente estudio confirman tal situación, toda vez que las imágenes del grupo OVX evidenciaron mayor cantidad de tejido adiposo de tipo unicelular en la médula de ósea al ser comparada con el grupo control.

El desarrollo de modelos que simulen un fenotipo osteoporótico, permiten en el área de la cirugía oral y maxilofacial, analizar la respuesta del hueso alveolar en sujetos con osteoporosis tanto en maxila como en mandíbula posterior a determinadas intervenciones clínicas, como exodoncias, instalación de implantes dentales, entre otros. No obstante, aun no existe consenso respecto de los tiempos necesarios para producir un fenotipo osteoporótico en maxila o mandíbula. En relación a esto Watanabe et al., 2020 en un estudio en ratas, indicaron que posterior a 2 meses de realizar la ovariectomía no observaron cambios morfológicos ni biomecánicos en el hueso mandibular. Mientras que Kim et al., 2020 a , en un estudio experimental en ratas, indicaron que el tiempo crítico para el deterioro de la regeneración del hueso alveolar fue posterior a 4 meses de realizada la ovariectomía. Haciendo parecer que el tiempo de espera posterior a la ovariectomía podría tener influencia en el desarrollo de este fenotipo osteoporótico .

Considerando las limitaciones del presente estudio, podemos concluir que a las 12 semanas post ovariectomía se observa un fenotipo histopatológico que sería compatible con características oseteoporóticas en ratas adultas. No obstante, nuevos estudios que permitan entender por ejemplo a nivel molecular como se va produciendo este cambio microarquitectónico del hueso, son necesarios.

PARRA, M.; ROMERO, I. \& OLATE, S. Descriptive analysis of an induced osteoporosis model in rats (Rattus norvegicus) (Rattus norvegicus). Int. J. Morphol., 39(1):282-286, 2021.

SUMMARY: The aim of the present study was to conduct a histopathological analysis of an animal model in rats, which simulates the characteristics observable in bones of patients with osteoporosis. To this end, 10 female rats (Rattus norvegicus) Sprague Dawley of 12 weeks of development and approximately 200-250 g were used. Of these, 5 underwent bilateral ovariectomy $(\mathrm{OVX})$, whereas the remaining 5 were used as control. After 12 weeks of ovariectomy in the experimental group, the animals were 
euthanized and the two femurs were collected, which were then sectioned to process their distal portion. All the animals survived at the end of the study without any signs of postoperative complications. In the experimental group (OVX), the histological images showed a decrease in the thickness of the cortical bone, a greater amount of cancellous bone, loss of the continuity of the periosteum and endostium around the bone matrix in addition to a greater amount of adipose tissue in the bone marrow, when compared with the control group. It can therefore be inferred that a histopathological phenotype can be found at 12 weeks postovariectomy that would be consistent with osteoporotic characteristics in adult rats.

Model

KEY WORDS: Osteoporosis; Ovariectomy; Animal

\section{REFERENCIAS BIBLIOGRÁFICAS}

Chen, C. H.; Wang, L.; Serdar Tulu, U.; Arioka, M.; Moghim, M. M.; Salmon, B.; Chen, C. T.; Hoffmann, W.; Gilgenbach, J.; Brunski, J. B. \& Helms, J. A. An osteopenic/osteoporotic phenotype delays alveolar bone repair. Bone, 112:212-19, 2018.

Gustafsson, A.; Wallin, M. \& Isaksson, H. Age-related properties at the microscale affect crack propagation in cortical bone. J. Biomech., 95:109326, 2019

Kim, H. J.; Kim, K. H.; Lee, Y. M.; Ku, Y.; Heo, S. J.; Rhyu, I. C. \& Seol, Y. J. Ovariectomy and timing of impaired maxillary alveolar bone regeneration: An experimental study in rats. J. Periodontol., 91(10):1357-66, 2020a.

Kim, H. N.; Xiong, J.; Macleod, R. S.; Iyer, S.; Fujiwara, Y.; Cawley, K. M.; Han, L.; He, Y.; Thostenson, J. D.; Ferreira, E.; Jilka, R. L.; Zhou, D.; Almeida, M. \& O'brien, C. A. Osteocyte RANKL is required for cortical bone loss with age and is induced by senescence. JCI Insight, 5(19): 138815, 2020b.

Klibanski, A.; Adams-Campbell, L.; Bassford, T. L.; Blair, S. N.; Boden, S. D.; Dickersin, K.; Gifford, D. R.; Glasse, L.; Goldring, S. R. \& Hruska, K. Osteoporosis prevention, diagnosis, and therapy. J. Am. Med. Assoc., 285(6):785-95, 2001.

Li, J.; Liu, X.; Zuo, B. \& Zhang, L. The Role of Bone Marrow Microenvironment in Governing the Balance between Osteoblastogenesis and Adipogenesis. Aging Dis., 7(4):514-25, 2016.

Park, S.; Heo, H. A.; Min, J. S. \& Pyo, S. W. Effect of Raloxifene on Bone Formation Around Implants in the Osteoporotic Rat Maxilla: Histomorphometric and Microcomputed Tomographic Analysis. Int. J. Oral Maxillofac. Implants., 35(2):249-56, 2020.

Raisz, L. G. Pathogenesis of osteoporosis: concepts, conflicts, and prospects. J. Clin. Invest., 115(12):3318-25, 2005.

Silveira, E. M. S.; Santos, M. C. Q.; Da Silva, T. C. B.; Silva, F. B. O.; Machado, C. V.; Elias, L.; Kolberg, A.; Kroth, A. \& Partata, W. A. Aging and low-intensity exercise change oxidative biomarkers in brain regions and radiographic measures of femur of Wistar rats. Braz. J. Med. Biol. Res., 53(6):e9237, 2020.

Tang, X.; Gao, Y.; Chen, Y.; Li, X.; Yu, P.; Ma, Z. \& Liu, R. Evaluation of the effect of $\mathrm{CaD}$ on the bone structure and bone metabolic changes in senile osteoporosis rats based on MLP-ANN methods. Food Funct., 10(12):8026-41, 2019.

Watanabe, K.; Lewis, S.; Guo, X.; Ni, A.; Lee, B. S.; Deguchi, T. \& Kim, D. G. Regional variations of jaw bone characteristics in an ovariectomized rat model. J. Mech. Behav. Biomed. Mater., 110:103952, 2020
Wei, B.; Huang, C.; Zhao, M.; Li, P.; Gao, X.; Kong, J.; Niu, Y.; Huang, R.; Quan, J.; Wei, J. \& Chu, J. Effect of Mesenchymal Stem Cells and Platelet-Rich Plasma on the Bone Healing of Ovariectomized Rats. Stem Cells Int., 2016:9458396, 2016.

Westbury, L. D.; Syddall, H. E.; Fuggle, N. R.; Dennison, E. M.; Cauley, J. A.; Shiroma, E. J.; Fielding, R. A.; Newman, A. B. \& Cooper, C. Long-term rates of change in musculoskeletal aging and body composition: findings from the Health, Aging and Body Composition Study. Calcif. Tissue Int., 106(6):616-24, 2020.

\author{
Dirección de Correspondencia: \\ Prof. Sergio Olate \\ Universidad de La Frontera \\ Claro Solar 115 \\ Temuco \\ CHILE
}

Email: sergio.olate@ufrontera.cl

Recibido: 07-07-2020

Aceptado: 20-09-2020 